Enfoques. Revista de Investigación en Ciencias de la Administración http://doi.org/10.33996/revistaenfoques.v2i7.38

No. 7 | Volumen 2 | Julio - Septiembre 2018 http://revistaenfoques.org ISSN: $2616-8219$

pp. $164-174$

\section{ESTRATEGIAS DE MERCADEO BASADAS EN EL MARKETING DIGITAL ORIENTADAS A LA CAPTACIÓN DE NUEVOS CLIENTES DE LAS PYMES. CASO DE ESTUDIO: GRUPO INTER GAME 2012, C.A.}

\author{
MARKETING STRATEGIES BASED ON DIGITAL MARKETING \\ AIMED AT ATTRACTING NEW CUSTOMERS OF SMES. CASE \\ STUDY: INTER GAME GROUP 2012, C.A.
}

\author{
Sandra Barón, Susana Fermín y Enmari Molina
}

\section{Resumen}

El presente artículo muestra un plan estratégico de mercadeo, basado en el marketing digital orientado a impulsar la captación de nuevos clientes de las PYMES, caso Grupo Inter Game 2012, C.A. Fue una investigación descriptiva con diseño de campo, realizándose el proceso de observación para la toma de la muestra y la aplicación de los instrumentos, los cuales estaban comprendidos por una entrevista estructurada y un cuestionario. Los datos recabados fueron analizados mediante índices estadísticos descriptivos individuales, mientras que para la entrevista se utilizó el análisis de contenido. La finalidad de la propuesta fue diseñar estrategias de marketing digital orientadas a la captación de nuevos clientes de Grupo Inter Game 2012, C.A. Se concluyó que la empresa desarrolla una labor satisfactoria para sus consumidores pero aún falta implementar estrategias que capten nuevos clientes y le permita no solo posicionarse en el mercado regional sino también nacional.

Palabras clave: Marketing digital; captación de clientes; PYMES; estrategias
Sandra Barón sbaron.19@gmail.com Universidad de Carabobo, Venezuela Licenciada en Contaduría Pública de la Universidad de Carabobo, Venezuela.

\section{Susana Fermín} inversiones2012@gmail.com Universidad de Carabobo, Venezuela Licenciada en Contaduría Pública de la Universidad de Carabobo, Venezuela.

\section{Enmari Molina}

enmarimolinasanchez@gmail.com Universidad de Carabobo, Venezuela

Licenciada en Contaduría Pública de la Universidad de Carabobo, Venezuela.

\begin{abstract}
This article shows a strategic marketing plan, based on digital marketing aimed at boosting the acquisition of new customers from SMEs, case Grupo Inter Game 2012, and C.A. It was a descriptive investigation with field design, performing the observation process for the sampling and the application of the instruments, which were comprised of a structured interview and a questionnaire. The data collected were analyzed using individual descriptive statistical indexes, while the content analysis was used for the interview. The purpose of the proposal was to design digital marketing strategies aimed at attracting new customers from Grupo Inter Game 2012 , C.A. It was concluded that the company develops a satisfactory task for its consumers but there is still a need to implement strategies that capture new customers and allow it to not only position itself in the regional market but also nationally.
\end{abstract}

Key words: Digital marketing; customer acquisition; SMEs; strategies

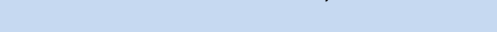



nuevos clientes de las Pymes. Caso de Estudio: Grupo Inter Game 2012, C.A.

\section{INTRODUCCIÓN}

Pudiera pensarse que con el boom de las tecnologías de información, los gerentes cuentan con información excesiva para tomar decisiones sobre bases reales, en ocasiones la cantidad, no hace la calidad y es preciso hacerse de estrategias adecuadas para que el producto pueda llegar al mercado meta, a la vez, recibir retroalimentación de la información necesaria para que las empresas logren satisfacer sus necesidades.

En la actualidad, una página web ya no es suficiente para atraer clientes en Internet y si una empresa necesita conseguir esto debe pensar seriamente en utilizar una estrategia de marketing digital. A diferencia de la publicidad tradicional donde la empresa es quien debe interrumpir y conseguir captar la atención del usuario, con el marketing digital es el potencial cliente quien toma la acción y la iniciativa de contactar a la empresa. La mayoría de las estrategias de marketing digital no requieren de una enorme inversión de dinero, pero sí de tiempo y dedicación.

Esta realidad destaca el auge de las tecnologías y la necesidad de comunicación en lo que hoy se conoce como Internet. Actualmente Internet se ha convertido en un instrumento clave en la informatización de las empresas, nivelando la manera de hacer negocios y aliviando los problemas tradicionales del mismo en desarrollo, como lo son la distancia que los separa de los mercados y la falta de inversión en infraestructura básica para el impulso de la economía, la ciencia, la cultura, la política y la educación.

Cada día se hace imprescindible el uso de la tecnología y sus diferentes medios para difundir y tener acceso a la información. A esto Carr (2011) indica que lo digital se ha convertido en la forma habitual de tratar la información por parte de los usuarios e Internet es ya una herramienta fundamental para buscar información, ver contenidos audiovisuales, comprar, relacionarse con otros, entretenerse o trabajar. En consecuencia, las estrategias de comunicación y publicidad de las empresas ya no pueden seguir siendo las mismas; por ende, ha surgido la necesidad de desarrollar planes estratégicos que les permitan adaptarse a dichos cambios y del mismo modo incrementar la competitividad haciendo uso de las diferentes herramientas de tecnologías de la información y comunicación disponibles.

A esta realidad, no escapan las Pequeñas y Medianas Empresas (PYMES) y dadas sus características particulares deben generar estrategias de adaptabilidad rápidas para no quedar rezagadas frente a la competencia. Por su parte las Pymes en América Latina se han ido incorporando a la nueva era digital, y es que en los últimos años esta región ha ido aumentando su participación en el uso de internet y sus diferentes medios. Rey (2012) señala que América Latina ha demostrado ser la región con el más rápido crecimiento de audiencia en internet, tomando en cuenta que es un mercado en donde la mayoría de los usuarios son personas jóvenes, de menos de 35 años, siendo Venezuela, Colombia y México los países con usuarios más jóvenes.

A pesar de su reciente creación en el año 2013 y de la incidencia de elementos externos como las condiciones sociopolíticas y la crisis económica nacional, que ha generado contracción en la mayoría de los mercados, la situación de la empresa Grupo Inter Game 2012, C.A, es considerada estable $y$ en crecimiento; sin embargo, surge por parte de sus directivos la necesidad de 
desarrollar nuevas estrategias que ayuden a incrementar las ventas y lograr posicionamiento en el mercado, tanto a nivel local como a nivel regional y nacional.

Es importante destacar que, a pesar de tener una visión de expansión de mercado, la empresa actualmente presenta las siguientes condiciones organizacionales como es la falta de definición e implementación de una estrategia de marketing efectiva, situación que trae como consecuencia rezago frente a la competencia en el mercado, un crecimiento lento (que pareciera ser solo respuesta de las necesidades de los consumidores y no de una estrategia de mercadeo), y un bajo conocimiento de la empresa entre los participantes del mercado. La empresa Grupo Inter Game 2012, C.A, sólo podrá mantenerse en el corto plazo en el mercado, ya que existen competidores mejor preparados y con estrategias de marketing definidas.

De allí que sea necesario utilizar el marketing digital, lo cual conllevaría a aumentar las ventas, fidelizar usuarios, posicionarse con respecto a otras empresas, integrar los diferentes canales de comunicación y crear un diálogo duradero con sus clientes. Además, la importancia del uso del marketing digital para las PYMES no radica en una moda o tendencia, radica en el cambio en la forma que el consumidor hoy en día se informa y toma decisiones de compra. Así como en los canales que utiliza para este proceso, a esto se suma que las ventas son cruciales para la existencia de un negocio y definitivamente el Marketing Digital le pudiera ofrecer la empresa Grupo Inter Game 2012, C.A., una plataforma parar mantener informados a sus clientes actuales, proporcionándoles ofertas adecuadas a sus necesidades y adicional a esto, poder llegar a clientes potenciales que no los conocen, ni han recibido información de sus productos y/o servicios.

En virtud de los planteamientos expuestos, se formuló la interrogante de la investigación que se resume en este artículo: ¿Qué estrategias de mercadeo basadas en el marketing digital podrán aplicarse en Grupo Inter Game 2012, C.A., para la captación de nuevos clientes de las PYMES? Planteándose para el estudio referido, como objetivo general: Proponer un plan estratégico de mercadeo, basado en el marketing digital orientado a impulsar la captación de nuevos clientes de las PYMES, caso Grupo Inter Game 2012, C.A. Y como objetivos específicos: Diagnosticar la situación actual de la empresa Grupo Inter Game 2012, C.A.; identificar los medios sociales utilizados por los consumidores; diseñar estrategias de marketing digital para la captación de nuevos clientes de Grupo Inter Game 2012, C.A.

$\mathrm{Se}$ ha querido iniciar esta parte introductoria señalando que la planificación estratégica constituye el marco referencial de acciones que permiten a las organizaciones desarrollar sus actividades, orientadas al alcance de metas y objetivos, tomando en consideración las características de la cultura organizacional de cada entidad. Al respecto, Fred (2003) señala que la planificación es "el arte y la ciencia de formular, implementar y evaluar decisiones multidisciplinarias que permiten que una empresa alcance sus objetivos" ( $s / n)$. En este sentido, se considera necesario la formulación de estrategias realistas que puedan ser aplicadas, esto no depende del tamaño de una empresa si no de tener bien definidos cuáles son sus objetivos.

Por consiguiente Amaya (2005) descompone las estrategias en los siguientes niveles: Estrategia de Nivel corporativo, estrategia a nivel funcional y estrategia de 

nuevos clientes de las Pymes. Caso de Estudio: Grupo Inter Game 2012, C.A.

unidad de negocios. También señala que, la planificación estratégica habla de la oportunidad de posicionar una marca en el mercado a través de metas trazadas, esto mediante el análisis de la situación de una empresa, visión (a donde quieren llegar, porque quieren llegar, como se puede llegar), metas u objetivos que quieren ser alcanzados, posibles estrategias aplicables para llegar a los objetivos.

Por su parte, Fred (2013) define tres etapas de la planificación estratégica como son: formulación, implementación y evaluación de estrategias. Para este autor, es importante destacar que a la hora de realizar una planificación estratégica hay que detenerse, y visualizar un antes y un después de la historia empresarial de la organización; con esto, se hace referencia al pasado de la empresa (dónde estuvo, qué hizo, qué funcionó y qué no) y la meta a la cual desea llegar a corto, mediano o largo plazo.

No obstante, hay que tener presente que una planificación estratégica no va a resolver la totalidad de las incertidumbres que puedan presentarse en una empresa, pero sí es una herramienta de cuantiosa importancia y de gran utilidad a la hora de replantear cualquier situación interna o externa de la cual se pueda obtener un beneficio, logro o avance.

En otro orden de ideas, Martínez (2006) define que el marketing es el conjunto de actividades dirigidas a la creación de los mercados o a la consecución de participaciones en mercados ya existentes, satisfaciendo las necesidades de los consumidores con determinados productos o servicios, obteniendo un beneficio para la empresa. En tal sentido, la rentabilidad de una empresa respaldada por el marketing va a depender de las actividades a desarrollarse, considerándose como una herramienta que deben conocer los empresarios para la obtención de un mayor patrocinio de un producto determinado, considerando así los siguientes principios de marketing para su desarrollo: Debe ir orientado hacia el consumidor; debe ser innovador; debe ser un sistema de valores; tiene que estar orientado hacia la sociedad.

Para enfocar el marketing, resulta de interés, hacer énfasis previamente en la definición de mercadeo, acotada por Kotler, y Armstrong (2003) como un proceso social y administrativo a través del cual individuos y los grupos obtienen lo que necesitan y desean mediante la elaboración, intercambio (oferta y demanda) para obtener un beneficio, o para satisfacer una necesidad.

Ahora bien, el marketing ha tenido cambios adaptados a los nuevos requerimientos del mercado $y$ consumidores; en este sentido la implementación de estrategias de marketing en las empresas supone un avance más en la superación del marketing tradicional. Este nuevo modelo, ofrece a los consumidores intervenir directamente en la definición de las estrategias empresariales y en la forma en que se relaciona con los clientes, través del uso de canales digitales y medios sociales, facilitando el acceso a la información requerida por los consumidores, generando valor agregado en cuanto a sus operaciones.

Al respecto, Kotler, Armstrong, Cámara y Cruz (2004), exponen que la Internet y otras tecnologías de información y cómputo han acrecentado considerablemente la capacidad de las empresas para realizar operaciones con mayor rapidez y precisión, dentro de un ámbito de tiempo y espacio mucho más amplio. Un sinnúmero de empresas ha establecido sitios Web para informar acerca de sus productos y servicios y promoverlos. 
De acuerdo con lo expuesto por los autores señalados, es evidente que el mercadeo digital conduce a mayor rapidez y bajo costo de las operaciones empresariales, contribuye a informar y promover los productos $y$ servicios que ofrece la empresa en el mercado, así como la rapidez al acceso de los bienes y/o servicios para los consumidores.

Por su parte, Santo (2014) plantea como beneficios del marketing digital lo siguiente: Ofrece a las pequeñas empresas competir con las más grandes en el mismo nivel; es más rentable que el marketing tradicional; es una estrategia efectiva ayuda a aumentar los beneficios para la empresa; facilita la interacción y proximidad con la audiencia; permite el acceso y conexión con los consumidores; y ayuda a generar confianza en la marca.

También es pertinente destacar que Dvoskin (2004) señala que la mezcla de marketing "es el resultado del conjunto de elecciones, acciones y decisiones de la organización respecto a cada una de las áreas; para responder a las necesidades que plantean distintos mercados, productos $y$ clientes. Así mismo, la necesidad de satisfacer las exigencias a la hora de presentar productos y servicios en el mundo digital, deriva la unión de varios elementos que conforman el mismo para llevar a cabo una planificación adecuada.

Con relación al posicionamiento, para García (2002) el posicionamiento está basado en el hecho de que los consumidores tienen una cierta percepción de los productos y las marcas. Estas percepciones se forman como consecuencia de las diferentes impresiones, sensaciones e informaciones que recibe del producto, ya sea a través de la publicidad, del precio, del envase, del vendedor, de otros consumidores o del consumo mismo. Señala además, que, el posicionamiento en el mercado se fundamenta en el lugar que tiene o toma un producto en la mente de los consumidores, de las ofertas que como empresa se ofrece a los clientes de manera que esta sea la seleccionada como primera opción dentro de su clase con respecto a otros competidores.

Por su parte, Kotler (2001) considera que el posicionamiento comienza con un producto: una mercancía, un servicio, una empresa, una institución o incluso una persona. Pero posicionamiento no es lo que se hace con el producto, posicionamiento es lo que se construye en la mente de las personas, es decir, se posiciona el producto en la mente del mercado meta.

\section{MATERIALES Y MÉTODO}

En torno al tipo de investigación, la misma fue concebida dentro de la modalidad general de estudio como una investigación diagnóstica descriptiva con diseño de campo. Es descriptiva, debido a que analiza e interpreta la situación actual de la empresa Grupo Inter Game 2012, C.A.

El método empleado tuvo una perspectiva cuantitativa, se fundamentó en la medición de las características de los fenómenos sociales, lo cual supone derivar un marco conceptual pertinente al problema analizado, una serie de postulados que expresen relaciones entre las variables estudiadas de forma deductiva. En tal sentido, se aplicó el método cuantitativo para presentar la información con la aplicación del cuestionario y la entrevista, para realizar el análisis de la población y muestra. En función a las características se determinó que la misma es de tipo no experimental, definida por Tamayo (2004). Como la investigación que se realiza sin manipular deliberadamente variables. Es 

nuevos clientes de las Pymes. Caso de Estudio: Grupo Inter Game 2012, C.A.

decir, se trata de estudios donde no hacemos variar de forma intencional las variables independientes para ver su efecto sobre otras variables. Lo que hacemos en la investigación no experimental es observar fenómenos tal y como se dan en su contexto natural, para después posteriormente analizarlos.

La población estuvo constituida por un (1) gerente general y ciento cincuenta (150) consumidores, cantidad que fue calculada tomando como referencia los clientes externos que asistieron al negocio por 10 días consecutivos, extrapolando esa cifra a un promedio mensual. Para desarrollar la investigación, se emplearon las técnicas de la encuesta, entrevista y la recopilación documental.

\section{RESULTADOS Y DISCUSIÓN}

Luego de haber aplicado el instrumento al gerente se analizó y sintetizó que la empresa realiza sus actividades acorde con su cultura organizacional, la cual se da a conocer a los empleados mediante la capacitación al momento de entrar en la organización lo que favorece positivamente en su desarrollo, se cuenta con inventario para satisfacer las demandas. Sin embargo, las políticas de mercadeo no han sido suficientemente buenas, es por ello que se requiere reformular las políticas implementadas.

Otro aspecto relevante es que la empresa cuenta con una buena ubicación en la zona central de Guacara, además de esto ofrece productos a precios competitivos. Pese a ello, no ha logrado obtener el nivel de ventas deseado motivado a la ausencia de estrategias de venta y desconocimiento del concepto y beneficios que brinda el marketing digital. Todos estos aspectos serán considerados para el diseño de un plan estratégico de mercadeo, basado en el marketing digital orientado a impulsar la captación de nuevos clientes para la empresa Grupo Inter Game 2012, C.A.

Por otra parte, se realizó un cuestionario a los clientes, lo cual permitió recabar información para el diagnóstico y determinar cuáles son las necesidades de los consumidores e identificar los medios sociales utilizados por ellos.

De los sesenta (60) clientes encuestados, el $80 \%$ de ellos oscila entre 31 y 45 años, los demás entre 23 y 30 años de edad, de ese $80 \%$ de clientes el $40 \%$ son empresarios, el $32 \%$ profesionales independientes $\quad 28 \% \quad$ profesionales asalariados. Con respecto al tipo de clientes, en su mayoría son frecuentes $67 \%$, un $15 \%$ de tendencia esporádica y $18 \%$ representado por quienes visitaban la tienda por primera vez. Siendo su motivo de visita al comercio un $33 \%$ la compra urgente, $29 \%$ búsqueda de presupuesto y $38 \%$ la curiosidad en los equipos de computación y electrónica como laptops, televisores y transmisores de imagen, computadores personales que allí se comercializan.

De los resultados obtenidos en la aplicación de los instrumentos de recolección de información, se resumen los siguientes aspectos de la empresa: el gerente de la misma afirma que el establecimiento comercial de la empresa está bien ubicado geográficamente; poseen un personal capacitado para desarrollar un buen proceso de ventas en conexión con la cultura organizacional; la empresa identifica tener una orientación hacia el cliente; los productos comercializados son de alta calidad; poseen un nivel de inventarios adecuados.

Por su parte, los consumidores estuvieron de acuerdo en su mayoría con 
que la empresa tiene variedad en los productos; marcas de las preferencias de los consumidores, las cuales son Hewlett Packard (HP) y Siragón; las condiciones del establecimiento de la empresa son adecuadas.

Como se evidencia en la presentación de los resultados, un $\mathbf{7 7 \%}$ los clientes conocen los medios sociales, y además la mayoría de los consumidores han utilizado los medios sociales (96\%) por tanto es un buen indicio para que la empresa aproveche esta circunstancia para desarrollarse mediante ellos y darse a conocer a través de dichos medios. De las personas que utilizan los medios sociales, el $73 \%$ han utilizado las redes sociales, un $16 \%$ han utilizado los Blogs, y un $11 \%$ de los encuestados dijo haber utilizado apps. De acuerdo a los resultados obtenidos y con base a los encuestados que utilizan redes sociales, se obtuvo que el $94 \%$ hace seguimiento por redes sociales de al menos una empresa, lo que permite conocer que un considerable número de consumidores suelen tener interacción en redes sociales con diferentes empresas, lo cual permite considerar la creación de cuentas en diferentes redes sociales como estrategias a utilizar.

La mayoría de los encuestados considera importante la presencia de las empresas en redes sociales y solo un $6 \%$ opina lo contario. En función a los resultados obtenidos se considera que es fundamental la presencia de las empresas en las redes sociales debido a que estas se han convertido en una gran herramienta para tener una mejor interacción con los clientes o usuarios y poder ofrecer los productos. Por otra parte, el $57 \%$ de los encuestados expresa que no han visitado alguna página web Empresarial. Se observa en conclusión, que hay bastante aceptación por la utilización de los medios y que a pesar que es un canal muy conocido, hay más experiencia en la utilización y seguimiento de las empresas a través de las redes, que la consulta que hacen ellos a una web empresarial.

Los consumidores manifestaron que la característica que más se aprecia observar en una página web, por parte de las empresas, es la realización de Promociones y/o Concursos de forma periódica, seguido de la exposición de marcas y especificaciones de los productos y/o servicios, y finalmente también están interesados en conocer precios de los productos.

Un considerable porcentaje de usuarios está a favor de tener acceso a la información mediante portales web, ya que eso facilitaría la difusión de información sobre los productos que ofrece la empresa.

Por último a los consumidores les interesaría encontrar en una estrategia de promoción: En un 50\% Información sobre descuentos, en un $20 \%$ Información sobre nuevos productos y características de productos existentes $y$ en un $10 \%$ participación en concursos. En términos generales, se pudo determinar cuáles son las necesidades, que en términos de información demandan los consumidores de los productos ofrecidos por la empresa.

\section{Propuesta de estrategia de marketing digital}

Después de analizar los resultados y realizar el diagnóstico estratégico de la empresa a través de la matriz Debilidades Oportunidades Fortalezas y Amenazas (DOFA), y reunir una serie de información concerniente a las necesidades de los consumidores y su receptividad a la utilización de medios sociales, se presenta a continuación como propuesta, un plan estratégico de mercadeo, basado en el 

nuevos clientes de las Pymes. Caso de Estudio: Grupo Inter Game 2012, C.A.

marketing digital orientado a impulsar la captación de nuevos clientes de Grupo Inter Game 2012, C.A. la cual teniendo un mercado muy amplio que cubrir pero con mucha competencia no ha logrado darse a conocer en el mercado con su variedad de productos. Con esta propuesta se buscó la orientación hacia las oportunidades económicas que contribuyan a su crecimiento.

Su objetivo general se puede entender como: Diseñar estrategias de marketing digital orientadas a la captación de nuevos clientes de Grupo Inter Game 2012, C.A. Y los objetivos específicos en términos de: Mantener a los consumidores actuales y aumentar el número de clientes de la empresa Grupo Inter Game 2012 C.A; incrementar el reconocimiento de la empresa a nivel sectorial, regional y nacional (posicionamiento); lograr el acercamiento hacia a sus clientes a través de una comunicación que permita la retroalimentación; conseguir que la información de la empresa, productos y servicios llegue a un mayor número de consumidores; e incrementar las ventas de la empresa Grupo Inter Game 2012 C.A, en un $20 \%$, que hayan sido influenciadas por el entorno online.

\section{Factibilidad de la propuesta}

Está compuesta por los recursos operativos, técnicos y económicos, que son necesarios para alcanzar los objetivos propuestos. El gerente de la empresa Grupo Inter Game 2012, C.A manifestó, interés y disposición en que la propuesta realizada sea aplicada, tomando en consideración los requerimientos necesarios para el desarrollo de la misma y de igual forma evaluando medidas que le permitan al personal adaptarse con tranquilidad y apoyo a las nuevas tecnologías.

La implementación de esta propuesta en la empresa no requiere mayor cantidad de equipos y materiales, puesto que el principal equipo requerido es un equipo tecnológico representado por un computador con una óptima conexión a la red, que brinde la posibilidad de llevar a cabo la ejecución de la propuesta.

Económicamente, la propuesta es viable para la empresa Grupo Inter Game 2012, C.A. dado que el gerente manifestó la disponibilidad económica para realizar efectuar los gastos necesarios que permitan aplicar las estrategias de marketing digital.

Es de vital importancia que toda empresa cuente con estrategias orientadas a la mejora y crecimiento constante, tomando en cuenta sus debilidades, fortalezas, amenazas y oportunidades, en este sentido luego de haber realizado el diagnóstico respectivo y el análisis situacional a través de la matriz DOFA, se presentan a continuación las estrategias planteadas, las cuales están sustentadas en las 7Ps del marketing digital.

Tomando como referencia los resultados obtenidos, se propone dirigir las estrategias a empresarios y profesionales con cierto poder de compra y que toman decisiones con respecto a sus necesidades familiares y empresariales. Estos consumidores en su mayoría son personas jóvenes en edades comprendidas entre 31 y 45 años, edad donde además de ser económicamente productivas, les gusta estar al día con los avances tecnológicos.

- Estrategias de posicionamiento: Dar a conocer la empresa Grupo Inter Game 2012, los productos y/o servicios a través de diferentes medios sociales más utilizados por los consumidores (redes 
sociales y pagina web). Generar contenido diferenciadores respecto a la competencia (promociones, servicio y atributos de los productos). Establecer alianzas con otras empresas de asesoría, para la publicación de Banners alusivos a la empresa Grupo Inter Game 2012, C.A.

- Estrategias de marketing Mix: Aumentar la capacidad informativa de los productos (especificaciones técnicas), modelos, marcas y servicios que ofrece la empresa. Adecuar el nivel de inventario en función de reponer eficientemente los productos más demandados. Generar confianza en los productos que no sean de marcas reconocidas. Mejorar la ubicación de los productos para una mejor exhibición de las marcas.

- Precio: Establecer alianzas con proveedores nacionales que faciliten la adquisición de productos de diferentes marcas. Definir precios competitivos respecto a la competencia. Ofrecer descuentos por suscripción a la página Web.

- Plaza: Establecer contacto con el cliente de manera más rápida por la ubicación del establecimiento comercial. Relacionar el establecimiento físico de la empresa con la información dada a conocer a través de la página web.

- Promoción: Indicar una sección de preguntas frecuentes en la página web. Crear promociones, descuentos y/o concursos para mejorar las ventas y dar a conocer esta información a través de la página web y los medios sociales. Crear un programa de afiliación, que genere incentivo a los consumidores.
- Personas: Definir la persona encargada del manejo de la página web y los medios sociales (Community Managger). Establecer criterios para el uso de las plataformas creadas que permitan dar a conocer la empresa a un mayor número de consumidores.

- Estrategias de contenido: Dar a conocer marcas y productos que añada valor agregado a los canales digitales de información utilizados.

- Estrategias de comunicación: Define cómo los visitantes son llevados al sitio web desde otros sitios web o a través de comunicaciones en otros canales. Traer visitantes a un sitio web desde otros sitios web (medios sociales), con el fin de establecer comunicación con las audiencias a través de medios digitales para lograr los objetivos de negocio. Atraer visitantes al sitio web o conseguir conocimiento de marca en sitios de terceros. Comunicarse con los segmentos a través de la publicidad interactiva, marketing en buscadores, patrocinios, acuerdos con terceros, email y marketing viral. Trasladar al sitio web desde otros medios no digitales con los segmentos objetivos a través de marketing directo en el establecimiento físico de la empresa, comunicando nuestra propuesta de valor online. Utilizar el canal online para conseguir ventas en canales offline. Comunicar promociones de primera compra. Optimización del diseño del sitio web, actualizando el contenido constantemente.

\section{CONCLUSIONES}

En la investigación que tuvo por objetivo general: Proponer un Plan Estratégico de 

nuevos clientes de las Pymes. Caso de Estudio: Grupo Inter Game 2012, C.A.

Mercadeo, basado en el marketing digital orientado a impulsar la captación de nuevos clientes de las PYMES en la empresa Grupo Inter Game 2012, C.A., en el mercado de productos y servicios de computación; se puede concluir que dicha empresa desarrolla una labor satisfactoria para sus consumidores pero aún falta implementar estrategias que aporten la captación de nuevos clientes y posicionarse en el mercado a pesar de la amplia competencia a la que se enfrenta.

Grupo Inter Game 2012, C.A. con sus productos y servicios, puede posicionarse no solamente en todo el Estado Carabobo sino también a nivel Nacional. El conocimiento de las herramientas de comunicación digital por parte de los clientes es una ventaja en la actualidad ya que por tratarse de una población grande que hace uso de ella con frecuencia, se convierten en consumidores potenciales que pudieran fidelizarse a la empresa por medio de estrategias de Marketing digital. Lo que permite concluir que el target al que va dirigido los productos y servicios de la empresa, son personas jóvenes y profesionales.

Existe por otra parte, la disposición del gerente para implementar el Marketing digital ya que cuentan con los recursos necesarios para lograrlo. Esto significa que la principal herramienta estratégica, se manifiesta en la utilización de marketing para conducir con éxito la actividad que realizan, y captar nuevos clientes, es decir, vincular el marketing digital como poderosa herramienta en el incremento de sus ventas y de la imagen de la empresa relacionándola con productos de marcas reconocidas y calidad de servicio, además, una retroalimentación con los clientes requerida para garantizar una óptima satisfacción de la demanda, aprovechando el recurso del internet, con grandes posibilidades de agilizar procesos a través del comercio electrónico. Todo esto en armonía con los objetivos planteados por la organización y su cultura organizacional, no sin antes haber realizado el diagnostico situacional de la empresa como base de partida en la generación del plan.

Otro de los objetivos alcanzados por la investigación fue determinar las necesidades de información de los consumidores, esto como insumo necesario para las decisiones de contenido y diseño de la página web y medios sociales. La investigación demuestra que los consumidores conocen y utilizan en su mayoría los medios digitales, razón por la cual se hace factible la implementación de la estrategia.

Finalmente, el diseñar estrategias de marketing digital para la captación de nuevos clientes de Grupo Inter Game 2012, C.A. se convierte en un aporte a la organización beneficiando su permanencia en el mercado. Por esta razón, el mercado digital, según lo expuesto por Peinado y Prado (2012), "se convierte en una oportunidad para que las empresas constituyan, entre muchas otras cosas, redes de valor accediendo a multitud de servicios optimizando sus procesos de aprovisionamiento" ( $\mathrm{s} / \mathrm{n})$. Las redes de valor son consideradas como una colaboración integrada de empresas especializadas en la que cada una ofrece servicios y bienes complementarios, enlazados por sofisticados sistemas de la información con el fin de crear y comercializar productos y servicios finales para el cliente.

Entre las bondades que ofrece el mercado digital se encuentra, primordialmente el factor de competitividad dentro de la economía. Estos mercados surgen como consecuencia de la búsqueda de economías de escala por parte de las 
empresas, debido a que, simplifican en gran parte el trabajo que realiza un comprador con sus proveedores. Entre estas tareas se encuentran por ejemplo, la creación y mantenimiento de catálogos, la formación de proveedores en los nuevos procesos, la conexión por parte de proveedores a una única aplicación o el mantenimiento de una infraestructura tecnológica, lo cual va a favorecer a los clientes actuales y a los futuros al satisfacer sus necesidades como consumidores.

\section{REFERENCIAS}

Amaya, J. (2005). Gerencia Planeación\& Estrategia. Colombia. Publicación por La Universidad Santo Tomas. Bucaramanga

Carr, N. (2011) Superficiales. ¿Qué está haciendo internet con nuestras mentes? Editorial W.W. Norton \& Company. Versión español Editorial Taurus

Dvoskin, R. (2004). Fundamentos de Marketing: Teoría y experiencia. 10 Edición. Buenos Aires, Argentina. Ediciones Granica, S.A

Fred R. (2003) Conceptos de Administración Estratégica. México. Editorial Pearson/Pentrice Hall

Fred R. (2013). Conceptos de Administración Estratégica. México. Editorial Pearson/Pentrice Hall. 14a Edición
García, H. C. (2004). Dirección de productos y marcas (Vol. 23). Editorial UOC

Kotler, P., Armstrong G., Cámara D. y Cruz I. (2004). Marketing. México. Editorial Prentice Hall

Kotler, P, y Armstrong, G. (2003) Fundamentos de Marketing. México. Pearson Educación

Kotler, P. (2001) Dirección de Mercadotecnia. Análisis, Planeación, Implementación y Control, México 8a Edición. Pearson Educación

Martínez, M. (2006). Gran Manual de Magnitudes Físicas y sus Unidades. Madrid, España. Ediciones Díaz de Santos S.A

Peinado, V. y Prado, R. (2012). Propuesta de una guía general de acción para la aplicación del marketing digital en las pequeñas y medianas empresas (PYMES), en la ciudad de Cumaná, Estado Sucre. Trabajo Especial de Grado no publicado, Universidad de Oriente, Cumaná

Rey, N. (2012). Marketing Digital. 8va Edición. España. Editorial Conde Nast

Santo, O. (2014). Guía Práctica de Marketing Digital para Pymes. España: Editorial Castilla

Tamayo y Tamayo, M. (2004). El proceso de investigación científica: incluye evaluación y administración de proyectos de investigación. 4a Edición. México: Limusas 